\title{
Spectral Properties of One Dimensional Quasi-Crystals
}

\author{
J. Bellissard ${ }^{1,2}$, B. Iochum ${ }^{1,2}$, E. Scoppola ${ }^{3}$, and D. Testard ${ }^{2,4}$ \\ ${ }^{1}$ Université de Provence, Marseille \\ ${ }^{2}$ Centre de Physique Theorique, CNRS, Luminy Case 907, F-13288 Marseille Cedex 9, France \\ ${ }^{3}$ Dip. Fisica, Università di Roma "La Sapienza", Ple A. Moro 2, I-00185 Roma, Italy \\ ${ }^{4}$ Université d'Aix, Marseille 2, Luminy
}

\begin{abstract}
In this paper we prove that the one dimensional Schrödinger operator on $l^{2}(\mathbb{Z})$ with potential given by:

$$
v(n)=\lambda \chi_{[1-\alpha, 1[}(x+n \alpha) \quad \alpha \notin \mathbb{Q}
$$

has a Cantor spectrum of zero Lebesgue measure for any irrational $\alpha$ and any $\lambda>0$. We can thus extend the Kotani result on the absence of absolutely continuous spectrum for this model, to all $x \in \mathbb{T}$.
\end{abstract}

\section{Model and Results}

In this paper we consider the discrete one dimensional Schrödinger hamiltonian with quasi-periodic potential, acting on $l^{2}(\mathbb{Z})$ and given by:

$$
H(x, \alpha, \lambda, I) \psi(n)=\psi(n+1)+\psi(n-1)+\lambda v(n, x) \psi(n),
$$

where

$$
v(n, x)=\chi_{\mathbf{I}}(n \alpha+x), \quad \lambda \in \mathbb{R} .
$$

$\chi_{\mathrm{I}}$ is the characteristic function of the interval $I$ in the torus $[0,1]$ : T. $I$ will be chosen equal to $[1-\alpha, 1[C \mathbb{T}, \alpha$ is a fixed irrational number in $[0,1]$.

Quasi periodic structures play an important role in solid state physics [J.D, S.O], in particular after the experimental discovery of quasi-crystals, that is of a metallic solid (an Al-Mn alloy) [Sh.Bl.Gr.Ca.] diffracting electrons like a crystal but with symmetry properties inconsistent with lattice translations. Schrödinger operators with quasi-periodic potentials taking only a finite number of values, like (2), can be used for instance to describe the properties of quasi-crystals.

In [Lu.Pe.] Luck and Petritis considered the phonon spectra in a one dimensional quasi-crystal. An almost periodic tiling of the line is constructed through a cut and projection method of a periodic 2-dim. structure on a one dimensional straight line with an irrational slope and the e.v. equation for the Laplace operator defined on this one dimensional almost periodic lattice, can be 
reduced exactly to Eq. (1) (see also [Be.] for references on quasicrystals and on almost periodicity in solid state physics). Moreover this kind of model seems to appear as an effective 1 electron hamiltonian in the description of the Peierls instability [Ma.Na.] at least in a mean field theory. The effective potential found by Machida-Nakano turns out to be discontinuous and qualitatively close to the model (1), in contrast with Aubry's choice $v(n, x)=2 \cos 2 \pi(n \alpha+x)$ [A.A]. The overall band structure obtained in that way looks very similar to that given in [Os.Kim]. Note in particular that there is no transition from a pure point spectrum when $\lambda$ varies.

Model (1), at last, provides a good example of a system with an exact renormalization group. This has been found first in Jacobi matrices associated with the Julia set of a polynomial [Be.B.M, BGH], the Laplacian of a Sierpinsky gasket without [Ra.] or with a magnetic field [Al, G.W.R.P.B, T.L.R], in the hierarchical model [J-L.Ma.Sc, H.K, K.L.S], with selfsimilar sequences generated by substitutions [Lu, A.A.K.M-F.P]. In this case an exact renormalization group transformation can be provided through transfer matrices.

Model (1) has been extensively investigated numerically and rigorously.

A lot of physical properties have been investigated for a one dimensional quasiperiodic lattice: electronic and phonon properties [K.B], localization of photons in optical layers [K.S.I], electrical resistance [S.K].

As far as the nature of the spectrum is concerned, we recall first of all [De.Pe.] where the absence of localization is proved for each $\lambda$ and for Lebesgue almost $\alpha$ and $x$, and where the characteristic function is taken over every interval $I \subset[0,1]$. (See also [Sü] for the case $\alpha=\frac{\sqrt{5}-1}{2}$ not contained in the set of zero measure of [De.Pe.].)

More recently in [Ko] a more general case is discussed, including the random potential given by independent identically distributed random variables, and the absence of absolutely continuous spectrum is proved for almost every $x$ and for every irrational $\alpha$. Combining these two results the spectrum of $H(x, \alpha, \lambda)$ turns out to be purely singular continuous for a.e. $x$ and $\alpha$.

As far as the structure of the spectrum of $H$ as set is concerned, there are a lot of numerical and rigorous papers concerning the golden case where $\alpha=\frac{\sqrt{5}-1}{2}$ and $I=\left[-\alpha^{3}, \alpha^{2}[\right.$. In [Ka.Ko.Ta.] (see also [O.P.R.S.S, R.O.S.S, K]) the relations for the transfer matrices and their traces are given on the Fibonacci sequence of numbers. In [G.A, G.A1] the relations for the transfer matrices are obtained for $\alpha$ equal to the silver and bronze mean and the model is also analyzed numerically. In [Kal.Ki.Le] recursive relations are given in the general case and the Hausdorff dimension of the spectrum is estimated. In [Ost.Kim] numerical computations of the spectrum are given for $I=(0, \alpha)$ and $\alpha$ varying through 1024 rational values, related to the binary Farey address which is used in that paper instead of the usual fraction representation.

More recently the golden case has been rigorously investigated by Casdagli [Ca]. Using symbolic dynamics techniques he proves that the dynamical spectrum

$$
B_{\infty} \equiv\left\{E \in \mathbb{R} ;\left|\operatorname{tr} M_{q_{n}}\right| \text { is bdd as } n \rightarrow \infty\right\},
$$


(where $M_{q_{n}}$ denotes the product of the first $q_{n}$ transfer matrices and $q_{n}$ is the Fibonacci sequence $\left.q_{n+1}=q_{n}+q_{n-1} q_{1}=1=q_{0}\right)$ is a Cantor set for $\lambda \geqq 16$. After this paper Süto proved that for the same model ( $\alpha$ : golden, $\left.I=\left[-\alpha^{3}, \alpha^{2}\right]\right)$ the spectrum of $H$ coincides with the dynamical spectrum $B_{\infty}$ and it is a Cantor set for $\lambda \geqq 4$. The proof given by Süto is "soft," involving only standard arguments in functional analysis.

We want also to mention the recent paper by Luck $[\mathrm{Lu}]$ studying the location of gaps and the dependence of gap widths on the potential strength.

In this paper we prove the following:

Theorem. $\forall$ irrational $\alpha$, let $I \equiv[1-\alpha, 1[, \forall \lambda>0, \forall x \in \mathbb{T}$,

$$
B_{\infty}=\sigma(H(x, \alpha, \lambda, I))=\{E \in \mathbb{R} ; \gamma(E)=0\},
$$

where $\sigma(H)$ denotes the spectrum of $H$ and $\gamma(E)$ is the Lyapunov exponent related to $H$.

Corollary. $\forall$ irrational $\alpha, \forall \lambda>0, \forall x \in \mathbb{T}, \sigma(H(x, \alpha, \lambda, I))$ is a Cantor set of zero Lebesgue measure.

Remark. This result can also be applied to extend to all $x \in \mathbb{T}$ the result of Kotani on the absence of absolutely continuous spectrum in this quasi-periodic case; for instance Kotani asks in his paper if the spectrum in the golden case with $x=0$ is sing. cont. $\forall \lambda>0$. We can answer yes. However our result seems to be insufficient in order to extend to all $x$ the absence of localization because we cannot exclude polynomially bounded eigenfunctions.

The proof of our theorem is based upon the following idea: for each irrational $\alpha$ and for $x=0$ it is possible to find explicitly recursive relations for the transfer matrices. With these relations it is possible to extend the results of Süto to each $\alpha$ using quite the same ideas, obtaining the first identity of the theorem. As far as the second identity is concerned the non-trivial problem is to prove that

$$
\{E \in \mathbb{R} ; \gamma(E)=0\} \supset B_{\infty}=\sigma(H(x, \alpha, \lambda, I)),
$$

that is, to show that the transfer matrices with exponentially growing norm cannot have bounded traces satisfying suitable recursive relations. In fact the following is a delicate point: from standard results the positivity of Lyapunov exponent implies exponential growth of the norm of transfer matrices only for a set of full Lebesgue measure of values of $x$ in $\mathbb{T}$. In particular, $x=0$ does not necessarily belong in this set. To solve this problem we needed a very detailed analysis, involving number theory, of the realization of the potential for arbitrary $x$.

Once the theorem is proved the corollary easily follows from the second part of the theorem and the following Kotani result [Ko.]:

$$
|\{E \in \mathbb{R} ; \gamma(E)=0\}|=0,
$$

where $|\cdot|$ denotes the Lebesgue measure.

In Sect. 2 we recall the main definitions and results related to number theory while in Sect. 3 we extend to all irrationals $\alpha$ the results given by Süto and prove the first identity of the theorem. In Sect. 4 we prove the second part of the theorem, and in Sect. 5 we briefly discuss some open problems. 


\section{Periodic Approximations}

Let us consider the expansion of $\alpha$ in continued fraction (see e.g. [La]):

$$
\alpha=\frac{1}{a_{1}+\frac{1}{a_{2}+\frac{1}{\cdots}}},
$$

where $a_{1}=\left[\frac{1}{\alpha}\right],[\cdot]$ denotes the integer part

$$
a_{i}=\left[\frac{1}{G^{(i+1)}(\alpha)}\right],
$$

and $G(\alpha)=\frac{1}{\alpha}-\left[\frac{1}{\alpha}\right]$.

In order to construct the rational approximations of $\alpha$, we define:

$$
\begin{array}{cc}
q_{n+1}=a_{n+1} q_{n}+q_{n-1} & q_{0}=1, q_{1}=a_{1}, \\
p_{n+1}=a_{n+1} p_{n}+p_{n+1} & p_{0}=0, p_{1}=1 .
\end{array}
$$

It is well known that (see [La])

$$
\begin{gathered}
\left|\alpha-\frac{p_{n}}{q_{n}}\right| \leqq \frac{1}{q_{n} q_{n+1}}, \\
\|k \alpha\| \geqq\left\|q_{n} \alpha\right\| \quad \forall k \in\left[1, \ldots, q_{n+1}[,\right.
\end{gathered}
$$

where $\|x\|=\inf _{n \in \mathbb{Z}}|x+n|$ is the distance of $x$ to $\mathbb{Z}$.

Lemma 1. a) $v(n, 0) \equiv v(n)=[(n+1) \alpha]-[n \alpha] \forall n \in \mathbb{Z}, n \neq-1$.

b) $v\left(q_{n}+k\right)=v(k) \quad 1 \leqq k<q_{n+1}-1$.

c) $v(-n)=v(n-1) \quad n \geqq 2$.

Proof. a) $v(n)=1 \Leftrightarrow n \alpha-[n \alpha] \in[1-\alpha, 1[\Leftrightarrow \exists m \in \mathbb{N} ; m+1-\alpha \leqq n \alpha<m+1$ with $n \alpha<m+1<(n+1) \alpha$, i.e. $m=[n \alpha]$. On the other hand

$$
[(n+1) \alpha]-[n \alpha]=\left\{\begin{array}{l}
0 \\
1
\end{array}\right.
$$

In fact

$$
0 \leqq[(n+1) \alpha]-[n \alpha] \leqq(n+1) \alpha-[n \alpha]=\{n \alpha\}+\alpha<2,
$$

where $\{\cdot\}$ is the fractional part,

$$
[(n+1) \alpha]-[n \alpha]=1 \Leftrightarrow \exists m \in \mathbb{N} ; m+1-\alpha \leqq n \alpha<m+1 .
$$

b) From Eq. (6) and (7) and part a) we have:

$$
\begin{aligned}
v\left(q_{n}+k\right) & =[(q+k+1) \alpha]-\left[\left(q_{n}+k\right) \alpha\right]=\left[(k+1) \alpha+\left(q_{n} \alpha-p_{n}\right)\right]-\left[k \alpha+q_{n} \alpha-p_{n}\right] \\
& =v(k),
\end{aligned}
$$

since $q_{n} \alpha-p_{n}=(-1)^{n}\left\|q_{n} \alpha\right\|$ and by (7) $\|m \alpha\|>\left\|q_{n} \alpha\right\| \forall m<q_{n+1}, m \neq q_{n}$. 
c) Is an immediate consequence of a).

Let

$$
T(n, x, \lambda, E) \equiv\left(\begin{array}{cr}
E-\lambda v(n, x) & -1 \\
1 & 0
\end{array}\right)
$$

be the transfer matrix associated to $H$, that is

$$
\left(\begin{array}{c}
\psi_{E}(n+1) \\
\psi_{E}(n)
\end{array}\right)=T(n, x, \lambda, E)\left(\begin{array}{c}
\psi_{E}(n) \\
\psi_{E}(n-1)
\end{array}\right),
$$

where $\psi_{E}(n)$ is a solution of

$$
H(x, \alpha, \lambda, I) \psi_{E}(n)=E \psi_{E}(n) .
$$

We have $\operatorname{det} T(n, x, \lambda, E)=1$.

Let $T(n) \equiv T(n, 0, \lambda, E)$ and

$$
\begin{gathered}
M_{(n)} \equiv M_{q_{m}}(0, \lambda, E) \equiv T\left(q_{n}\right) \cdot \ldots \cdot T(2) \cdot T(1), \\
M_{(0)}=T(1)=\left(\begin{array}{rr}
E & -1 \\
1 & 0
\end{array}\right), \quad M_{(1)}=T\left(a_{1}\right) \cdot \ldots \cdot T(1) .
\end{gathered}
$$

\section{Proposition 1.}

$$
M_{(n+1)}=M_{(n-1)} M_{(n)}^{a_{n+1}} \quad \forall n \geqq 1 .
$$

The proof of this proposition is an easy consequence of Lemma 1b). By using the definition

$$
M_{(-1)} \equiv\left(\begin{array}{rr}
1 & -\lambda \\
0 & 1
\end{array}\right),
$$

Proposition (1) can be extended also to the case $n=0$. We remark at this point that the order from left to right in the values of the potential correspond to the order from right to left in the product of transfer matrices.

In the same way we can define

$L_{(n)} \equiv\left[T(1) \cdot \ldots \cdot T\left(q_{n}\right)\right]^{-1}$ and we have, by using Lemma 1c), and Proposition 1):

$$
\begin{array}{cc}
\psi\left(-q_{n}-2\right)=L_{(n)} \psi(-2) & n \geqq 1, \\
L_{(n+1)}=M_{(n+1)}^{-1}=L_{(n)}^{a_{n+1}} L_{(n-1)} & \forall n \geqq 1 .
\end{array}
$$

Lemma 2. For each $2 \times 2$ matrix $M$ with $\operatorname{det} M=1$ and $\forall a \in \mathbb{N}$ :

i) $M^{a}=S_{a-1}(\xi) M-S_{a-2}(\xi)$ II

with $\xi=\operatorname{tr} M$ and $S_{a}(\xi)$ the Chebyshev polynomials:

$$
S_{a}(\xi)=S_{a-1}(\xi) \xi-S_{a-2}(\xi), \quad S_{1}(\xi)=\xi, \quad S_{0}(\xi)=1, \quad S_{-1}(\xi)=0
$$

and for these polynomials, using the recursive relation, it is immediate to prove that the quantity $S_{a} S_{a-2}-S_{a-1}^{2}$ is independent of $a$, this is

$$
S_{a} S_{a-2}-S_{a-1}^{2}=\text { const }=S_{1} S_{-1}-S_{0}^{2}=-1 \text {. }
$$


ii) If $|\xi| \equiv|\operatorname{tr} M|>2$ then

$$
S_{a}(\xi)=(\operatorname{sgn} \xi)^{a} \frac{\operatorname{sh}(a+1) \theta}{\operatorname{sh} \theta} \forall a \geqq 1,
$$

where $\theta>0$ is such that $\xi= \pm 2 \operatorname{ch} \theta$. Moreover $S_{a}(\xi)<|\xi|^{a}$.

If $|\xi|=2$ then

$$
S_{a}(\xi)=(\operatorname{sign} \xi)^{a}(a+1) \forall a \geqq 1,
$$

if $|\xi|<2 \xi=2 \cos \theta, \theta \in(0, \pi)$ then

$$
S_{a}(\xi)=\frac{\sin (a+1) \theta}{\sin \theta} \forall a \geqq 1 \text {. In this case } S_{a}(\xi)<a+1 \text {. }
$$

Proof of Lemma 2. By induction on $a$ :

$$
\begin{gathered}
M^{2}+\mathbb{1}=M\left(M+M^{-1}\right)=M(\operatorname{tr} M), \\
M^{a+1}=S_{a-1}(\xi) M^{2}-S_{a-2}(\xi) M=\left[S_{a-1}(\xi) \cdot \xi-S_{a-2}(\xi)\right] M-S_{a-1}(\xi) \mathbb{1} .
\end{gathered}
$$

Point ii) can be easily verified using the recursive relations.

Proposition 2. $\forall n \geqq 1$ let $\xi_{n} \equiv \operatorname{tr} M_{(n)}$. If $\left|\xi_{n-1}\right|>2$ then

$$
\xi_{n+1}=\xi_{n} S_{a_{n+1}-1}\left(\xi_{n}\right) \frac{S_{a_{n}}\left(\xi_{n-1}\right)}{S_{a_{n}-1}\left(\xi_{n-1}\right)}-\xi_{n-1} S_{a_{n+1}-2}\left(\xi_{n}\right)-\xi_{n-2} \frac{S_{a_{n+1}-1}\left(\xi_{n}\right)}{S_{a_{n}-1}\left(\xi_{n-1}\right)} .
$$

Remark 1. We observe that in Lemma 4 we assumed $\left|\xi_{n-1}\right|>2$ in order to have $S_{a_{n-1}}\left(\xi_{n-1}\right) \neq 0$, while in the case $S_{a_{n-1}}\left(\xi_{n-1}\right)=0$ using Lemma 2 we have $M_{n-1}^{a_{n}}$ $=-S_{a_{n-2}}\left(\xi_{n-1}\right) \mathbb{1}=S_{a_{n}}\left(\xi_{n-1}\right) \mathbb{1}= \pm \mathbb{1}$, that is $\xi_{n}= \pm \xi_{n-2}$, and it is possible to find a relation between $\xi_{n+1}$ and $\xi_{n-2}, \xi_{n-3}$.

Remark 2. If $a_{n}=1 \forall n$, that is in the case of the golden number, Eq. (10) becomes:

$$
\xi_{n+1}=\xi_{n} \xi_{n-1}-\xi_{n-2}
$$

as in [Ka.Ko.Ta, Ca., Sü.].

Proof of Proposition 2. By Proposition 1 and Lemma 2:

$$
\begin{gathered}
M_{(n+1)}=M_{(n-1)} M_{(n)}^{a_{n+1}}=M_{(n-1)} M_{(n)} S_{a_{n+1}-1}\left(\xi_{n}\right)-M_{(n-1)} S_{a_{n+1}-2}\left(\xi_{n}\right), \\
M_{(n)}=M_{(n-2)} M_{(n-1)}^{a_{n}}=M_{(n-2)} M_{(n-1)} S_{a_{n-1}}\left(\xi_{n-1}\right)-M_{(n-2)} S_{a_{n}-2}\left(\xi_{n-1}\right), \\
M_{(n)} M_{(n-1)}=M_{(n-2)} M_{(n-1)}^{a_{a+1}}=M_{(n-2)} M_{(n-1)} S_{a_{n}}\left(\xi_{n-1}\right)-M_{(n-2)} S_{a_{n}-1}\left(\xi_{n-1}\right) .
\end{gathered}
$$

From (12)

$$
M_{(n-2)} M_{(n-1)}=\frac{M_{(n)}+M_{(n-2)} S_{a_{n}-2}\left(\xi_{n-1}\right)}{S_{a_{n-1}}\left(\xi_{n-1}\right)} .
$$

From (13) and (14)

$$
M_{(n)} M_{(n-1)}=S_{a_{n}}\left(\xi_{n-1}\right) \frac{M_{(n)}+M_{(n-2)} S_{a_{n}-2}\left(\xi_{n-1}\right)}{S_{a_{n}-1}\left(\xi_{n-1}\right)}-M_{(n-2)} S_{a_{n-1}}\left(\xi_{n-1}\right) .
$$


Taking the trace of (11) and using (15),

$$
\begin{aligned}
& \operatorname{tr} M_{(n+1)} \\
& \equiv \xi_{n+1}=\left[S_{a_{n}}\left(\xi_{n-1}\right) \frac{\xi_{n}+\xi_{n-2} S_{a_{n}-2}\left(\xi_{n-1}\right)}{S_{a_{n}-1}\left(\xi_{n-1}\right)}-\xi_{n-2} S_{a_{n}-1}\left(\xi_{n-1}\right)\right] S_{a_{n+1}-1}\left(\xi_{n}\right) \\
& \quad-\xi_{n-1} S_{a_{n+1}-2}\left(\xi_{n}\right)=\xi_{n} S_{a_{n+1}-1}\left(\xi_{n}\right) \frac{S_{a_{n}}\left(\xi_{n-1}\right)}{S_{a_{n-1}}\left(\xi_{n-1}\right)} \\
& \quad+\xi_{n-2} S_{a_{n+1}-1}\left(\xi_{n}\right)\left[\frac{S_{a_{n}-2}\left(\xi_{n-1}\right)}{S_{a_{n-1}}\left(\xi_{n-1}\right)} \cdot S_{a_{n}}\left(\xi_{n-1}\right)-S_{a_{n-1}}\left(\xi_{n-1}\right)\right] \\
& \quad+-\xi_{n-1} S_{a_{n+1}-2}\left(\xi_{n}\right)
\end{aligned}
$$

and by (9)

$$
\frac{S_{a_{n}-2}\left(\xi_{n-1}\right) S_{a_{n}}\left(\xi_{n-1}\right)}{S_{a_{n}-1}\left(\xi_{n-1}\right)}-S_{a_{n-1}}\left(\xi_{n-1}\right)=-\frac{1}{S_{a_{n-1}}\left(\xi_{n-1}\right)} .
$$

Proposition 3. Let $\left\{\xi_{n}\right\}_{1}^{\infty}$ be the sequence generated by (10) with

$$
\xi_{-1}=2, \quad \xi_{0}=E, \quad \xi_{1}=S_{a_{1}-1}(E)(E-\lambda)-S_{a_{1-2}}(E) 2 ;
$$

then the quantity:

$$
I_{n} \equiv \xi_{n+1}^{2}+\xi_{n}^{2}+\left[\operatorname{tr}\left(M_{(n)} M_{(n+1)}\right)\right]^{2}-\xi_{n+1} \xi_{n} \operatorname{tr}\left[M_{(n)} M_{(n+1)}\right]
$$

is constant in $n$ and

$$
I_{n}=\lambda^{2}+4
$$

Proof of Proposition 3. Using the identity

$$
\operatorname{tr}(A B)=\operatorname{tr}(A) \operatorname{tr}(B)-\operatorname{tr}\left(A B^{-1}\right)
$$

which holds for $2 \times 2$ matrices with $\operatorname{det} A=\operatorname{det} B=1$ (see Lemma 2), we can prove the following:

$$
I_{n}=\operatorname{tr}\left(M_{(n+1)}^{-1} M_{(n)}^{-1} M_{(n+1)} M_{(n)}\right)+2 .
$$

In fact using (17) three times:

$$
\begin{aligned}
\operatorname{tr}[ & \left.M_{(n+1)}^{-1} M_{(n)}^{-1} M_{(n+1)} M_{(n)}\right]=\left[\operatorname{tr}\left(M_{(n+1)} M_{(n)}\right)\right]^{2}-\operatorname{tr}\left(M_{(n)}^{2} M_{(n+1)}^{2}\right) \\
= & {\left[\operatorname{tr}\left(M_{(n+1)} M_{(n)}\right)\right]^{2}-\left\{\operatorname{tr}\left(M_{(n)}\right) \operatorname{tr}\left(M_{(n)} M_{(n+1)}^{2}\right)-\operatorname{tr}\left(M_{(n+1)}^{2}\right)\right\} } \\
= & {\left[\operatorname{tr}\left(M_{(n+1)} M_{(n)}\right)\right]^{2}+\operatorname{tr}\left(M_{(n+1)}^{2}\right) } \\
& -\operatorname{tr}\left(M_{(n)}\right)\left[\operatorname{tr}\left(M_{(n)} M_{(n+1)}\right) \cdot \operatorname{tr}\left(M_{(n+1)}-\operatorname{tr} M_{(n)}\right)\right] \\
= & {\left[\operatorname{tr}\left(M_{(n+1)} M_{(n)}\right)\right]^{2}+\left[\operatorname{tr}\left(M_{(n+1)}\right)\right]^{2}+\left[\operatorname{tr}\left(M_{(n)}\right)\right]^{2} } \\
& -\operatorname{tr}\left(M_{(n)}\right) \operatorname{tr}\left(M_{(n)} M_{(n+1)}\right) \cdot \operatorname{tr}\left(M_{(n+1)}\right)-2 .
\end{aligned}
$$

Moreover the recursive relation gives:

$$
\begin{aligned}
& \operatorname{tr}\left(M_{(n+1)}^{-1} M_{(n)}^{-1} M_{(n+1)} M_{(n)}\right)=\operatorname{tr}\left(M_{(n+1)} M_{(n)} M_{(n+1)}^{-1} M_{(n)}^{-1}\right) \\
& \left.\quad=\operatorname{tr} M_{(n-1)} M_{(n)}^{a_{n+1}+1}\left(M_{(n)}^{a_{n+1}}\right)^{-1} M_{(n-1)}^{-1} M_{(n)}^{-1}\right) \\
& \quad=\operatorname{tr}\left(M_{(n-1)} M_{(n)} M_{(n-1)}^{-1} M_{(n)}^{-1}\right)=I_{n-1}-2,
\end{aligned}
$$

thus $I_{n}=I_{n-1}=I_{0}=\lambda^{2}+4$. 
We remark that the quantity $I_{n}$ is also equal to

$$
I_{n}=\frac{1}{2} \operatorname{tr}\left(\left[M_{(n)}, M_{(n+1)}\right]^{2}\right)+4 .
$$

\section{The Spectrum of $H(x, \alpha, \lambda, I)$}

The study of the spectrum of $H(x, \alpha, \lambda, I)$ can be done following step by step Süto's paper. First we extend to right-continuous potentials the well known result of quasi-periodic hamiltonians about the independence of $x$, for all $x \in \mathbb{T}$, of the spectrum, as closed set, (see e.g. [Si]).

Lemma 3. For any $x, y$ in $\mathbb{T}$ we have $\sigma(H(x, \alpha, \lambda, I))=\sigma(H(y, \alpha, \lambda, I))$.

Proof of Lemma 3. Since $H(x+n \alpha, \alpha, \lambda, I)=T_{n}^{-1} H(x, \alpha, \lambda, I) T_{n}$, where $T_{n}$ is the translation by $n$ in $l^{2}(\mathbb{Z}) \sigma(H(x+n \alpha, \alpha, \lambda, I))=\sigma(H(x, \alpha, \lambda, I))$ for any $n \in \mathbb{Z}$, the potential being pointwise right-continuous in $x$, the Hamiltonian $H(x, \alpha, \lambda, I)$ is strongly right-continuous. Moreover if $A$ and $A_{m}$ are bounded selfadjoint operators on a Hilbert space such that $A=s-\lim A_{m}$, then $\sigma(A) \subset \sigma\left(A_{m}\right)$ for some large $m$ (see e.g. [Re.Si, p. 290]). $\alpha$ being irrational, there exists $\left(n_{k}\right)_{k} \in \mathbb{Z}$ such that $0 \leqq x+n_{k} \alpha-y \rightarrow 0$ when $k \rightarrow \infty$. Thus $\sigma(H(y, \alpha, \lambda, I)) \subset \sigma\left(H\left(x+n_{k} \alpha, \alpha, \lambda, I\right)\right)$ for large $k$ and $\sigma(H(y, \alpha, \lambda, I)) \subset \sigma(H(x, \alpha, \lambda, I))$.

We can then consider the case $x=0$ for which we have all the recursive relations on the traces of the transfer matrices shown in the previous section.

The only non-trivial point in the proof of the first identity in the theorem, is the proof of the following result analogous of Lemma 2 in [Sü.]:

Proposition 4. Let $\left\{\xi_{i}\right\}_{1}^{\infty}$ be the sequence generated by (10) with

$$
\xi_{-1}=2, \quad \xi_{0}=E, \quad \xi_{1}=S_{a_{1-1}}(E)(E-\lambda)-S_{a_{1-2}}(E) \cdot 2 .
$$

A sufficient and necessary condition that $\left\{\xi_{n}\right\}$ be unbounded is that for some $N \geqq 0$,

$$
\left|\xi_{N-1}\right| \leqq 2, \quad\left|\xi_{N}\right|>2, \quad\left|\xi_{N+1}\right|>2 .
$$

This $N$ is unique, $\left|\xi_{n+2}\right|>\frac{\left|\xi_{n+1}\right|\left|\xi_{n}\right|}{2}>2 \forall n \geqq N$ and there exists $C>1$ such that $\frac{\left|\xi_{n}\right|}{2}>C^{q_{n}}$. If $\left\{\xi_{n}\right\}$ is bounded then:

$$
\left|\xi_{n}\right|<2+\sqrt{8+\lambda^{2}}
$$

With this proposition the construction of Süto can be repeated exactly in the same way in order to show that the spectrum coincides with the set $B_{\infty}=\left\{E ;\left|\xi_{n}\right|\right.$ bdd $\}$, hence the first part of the theorem.

We do not give here all the details and we refer to the very clear Süto paper [Sü].

We want to remark at this point that it would be possible to extend also the proof of the Cantor spectrum for $\lambda>4$ using the same simple strategy as in [Sï]. However this strategy will definitively not work for $\lambda<4$, and we shall prove in the following section, by using different ideas, the stronger result on the Cantor spectrum, with $\lambda>0$. 
Proof of Proposition 4. Suppose (18) true for some $N \geqq 0$, then by (10) and by the relation:

$$
\left|\frac{S_{a_{N+1}}\left(\xi_{N}\right)}{S_{a_{N+1}-1}\left(\xi_{N}\right)}\right|=\frac{\operatorname{sh}\left(a_{N+1+1}\right) \theta_{N}}{\operatorname{sh} a_{N+1} \theta_{N}}=\operatorname{ch} \theta_{N}+\frac{\operatorname{ch} a_{N+1} \theta_{N}}{\operatorname{sh} a_{N+1} \theta_{N}} \operatorname{sh} \theta_{N}
$$

we have

$$
\begin{aligned}
&\left|\xi_{N+2}\right| \geqq\left|\xi_{N+1}\right|\left|S_{a_{N+2}-1}\left(\xi_{N+1}\right)\right|\left[\frac{1}{2}\left|\xi_{N}\right|+\frac{\operatorname{ch} a_{N+1} \theta_{N}}{\operatorname{sh} a_{N+1} \theta_{N}} \cdot \operatorname{sh} \theta_{N}\right] \\
&-\left\{\left|\xi_{N}\right|\left|S_{a_{N+2}-2}\left(\xi_{N+1}\right)\right|+\left|\xi_{N-1}\right|\left|\frac{S_{a_{N+2}-1}\left(\xi_{N+1}\right)}{S_{a_{N+1}-1}\left(\xi_{N}\right)}\right|\right\} \\
& \geqq\left|\xi_{N}\right|\left|\operatorname{ch} a_{N+2} \theta_{N+1}\right| \geqq \frac{\left|\xi_{N}\right|\left|\xi_{N+1}\right|}{2}
\end{aligned}
$$

since

$$
\operatorname{ch} \theta_{N+1} \frac{\operatorname{sh} a_{N+2} \theta_{N+1}}{\operatorname{sh} \theta_{N+1}}=\operatorname{ch} a_{N+2} \theta_{N+1}+\frac{\operatorname{sh}\left(a_{N+2}-1\right) \theta_{N+1}}{\operatorname{sh} \theta_{N+1}}
$$

and

$$
\begin{gathered}
2 \operatorname{ch} \theta_{N} \frac{\operatorname{sh}\left(a_{N+2}-1\right) \theta_{N+1}}{\operatorname{sh} \theta_{N+1}}+2 \operatorname{ch} \theta_{N+1} \frac{\operatorname{sh} a_{N+2} \theta_{N+1}}{\operatorname{sh} \theta_{N+1}} \frac{\operatorname{ch} a_{N+1} \theta_{N}}{\operatorname{sh} a_{N+1} \theta_{N}} \cdot \operatorname{sh} \theta_{N} \\
>2 \operatorname{ch} \theta_{N} \frac{\operatorname{sh}\left(a_{N+2}-1\right) \theta_{N+1}}{\operatorname{sh} \theta_{N+1}}+2 \frac{\operatorname{sh} a_{N+2} \theta_{N+1}}{\operatorname{sh} \theta_{N+1}} \frac{\operatorname{sh} \theta_{N}}{\operatorname{sh} a_{N+1} \theta_{N}} .
\end{gathered}
$$

From (19) by induction $\forall n>N$ we have $\left|\xi_{n+2}\right| \geqq\left|\xi_{n}\right|\left|\operatorname{ch} a_{n+2} \theta_{n+1}\right|>\frac{\left|\xi_{n}\right|\left|\xi_{n+1}\right|}{2}$ that is

$$
\frac{\left|\xi_{n+2}\right|}{2}>\frac{\left|\xi_{n}\right|}{2}\left(\frac{\left|\xi_{n+1}\right|}{2}\right)^{a_{n+2}} \text {, thus } \frac{\left|\xi_{n}\right|}{2}>C^{q_{n}} \text { with } C>1
$$

For $n=N$

$$
\left|\xi_{n-1}\right| \leqq 2<\left|\xi_{n}\right|, \quad\left|\xi_{n+1}\right|<\left|\xi_{n+2}\right|<\ldots
$$

Clearly these inequalities cannot hold for other values of $n$.

If $(18)$ doesn't hold for every $N \geqq 0$ this means that:

$$
\text { if }\left|\xi_{n}\right|>2 \text { then }\left|\xi_{n-1}\right| \leqq 2 \text { and }\left|\xi_{n+1}\right| \leqq 2 \text {, }
$$

otherwise we would get (18) for $N \leqq n$; in fact $\left|\xi_{-1}\right|=2$, let $n_{0} \equiv \min \{n \in \mathbb{N}$; $\left.n \geqq 0\left|\xi_{n}\right|>2\right\}$. This implies $\left|\xi_{n_{0}-1}\right| \leqq 2$ and by the opposite of (18) $\left|\xi_{n_{0}+1}\right| \leqq 2$ and so on for $n_{1} \equiv \min \left\{n \in \mathbb{N} ; n>n_{0}+1 ;\left|\xi_{n}\right|>2\right\}, n_{2}$, etc.... This argument proves that the opposite of (18) implies (20). We must now distinguish between two possibilities

i) (20) holds and $\left|\operatorname{tr}\left(M_{n} M_{n-1}\right)\right| \leqq 2$,

ii) (20) holds and $\left|\operatorname{tr}\left(M_{n} M_{n-1}\right)\right|>2$.

In case i) using Proposition (3)

$$
\begin{aligned}
I_{n-1} & \equiv \xi_{n}^{2}+\xi_{n-1}^{2}+\left[\operatorname{tr}\left(M_{n} M_{n-1}\right)\right]^{2}-\xi_{n} \xi_{n-1} \operatorname{tr}\left(M_{n} M_{n-1}\right) \\
& =\lambda^{2}+4 \geqq \xi_{n}^{2}-4\left|\xi_{n}\right|, \text { which implies }\left|\xi_{n}\right| \leqq 2+\sqrt{8+\lambda^{2}} .
\end{aligned}
$$


Case ii) is impossible since (11), (20) and $\left|\operatorname{tr}\left(M_{n} M_{n-1}\right)\right|>2$ imply:

$$
\text { if } \xi_{n}= \pm 2 \operatorname{ch} \theta_{n}, \theta_{n}>0 \quad\left|\xi_{n+1}\right|=\left|\operatorname{tr} M_{n+1}\right| \geqq 2 \frac{\operatorname{sh} a_{N+1} \theta_{N}}{\operatorname{sh} \theta_{N}}-2 \frac{\operatorname{sh}\left(a_{N+1}-1\right) \theta_{N}}{\operatorname{sh} \theta_{N}} \geqq 2,
$$

in contradiction with (20).

\section{Proof of the Second Part of the Theorem: $\sigma(H(x, \alpha, \lambda, I))=\{E \in \mathbb{R} ; \gamma(E)=0\}$}

The inclusion $\{E \in \mathbb{R} ; \gamma(E)=0\} \subset \sigma(H(x, \alpha, \lambda, I))$ is trivial, in fact let $E$ be an energy contained in the resolvent set of $H$, we know, by the Combes-Thomas argument (see e.g. [Ma.Sc.]) that the corresponding Green's function, solution of the equation $(H-E) G(x, y)=\delta_{x, y}$, decay exponentially:

$$
|G(x, y)|<\text { const. } \exp \{-m|x-y|\}, \quad \text { where } \quad m=\ln (c \operatorname{dist}(E, \sigma(H))+1) .
$$

This implies that the solution of the equation $(H-E) \phi=0$ with conditions $\phi(0)=1, \phi(-1)=0$ grows exponentially with strictly positive mass (since, for instance, the Wronskian is constant $W(G(x, 0), \phi(x))=$ const.). On the other hand we know that for almost all $x$

$$
\lim _{L \rightarrow \infty} \frac{1}{L} \ln \left\|M_{L}(x, \lambda, E)\right\|=\gamma(E)
$$

and

$$
|\phi(L)|^{2}<|\phi(L)|^{2}+|\phi(L+1)|^{2}<\left\|M_{L}(x, \lambda, E)\right\|^{2},
$$

that is $\gamma(E)>0$.

As far as the opposite inclusion is concerned, by using the first part of the theorem, we have to show that: for each irrational $\alpha \in \mathbb{T}, \forall \lambda>0$,

$$
B_{\infty} \subset\{E \in \mathbb{R} ; \gamma(E)=0\},
$$

where $\gamma(E)=\gamma(E, \lambda)$ is the Lyapunov exponent, that is: [see e.g. [Bou.Lac]]) for Lebesgue almost all $x \in \mathbb{T}$,

$$
\lim _{L \rightarrow \infty} \frac{1}{L} \ln \left\|M_{L}(x, \lambda, E)\right\|=\gamma(E) .
$$

We will prove that

$$
\{E ; \gamma(E)>0\} \subset B_{\infty}^{c} \text {. }
$$

Let us fix $E$ such that $\gamma(E)>0$, then by (21) there exists a set $\Omega_{0} \subset \mathbb{T}$ of Lebesgue measure $\left|\Omega_{0}\right|=1$ such that $\forall x \in \Omega_{0} \forall \varepsilon>0 \exists N(x, \varepsilon) ; \forall L>N(x, \varepsilon)$,

$$
e^{(\gamma(E)+\varepsilon) L} \geqq\left\|M_{L}(x, \lambda, E)\right\|>e^{(\gamma(E)-\varepsilon) L} .
$$

Lemma 4. $\forall$ irrational $\alpha, \forall n \exists A n \subset \mathbb{T}$ with

$$
\sum_{n=N}^{\infty}\left|A_{n}\right| \leqq \varepsilon(N) \text { and } \quad \varepsilon(N) \rightarrow 0 \text { as } \quad N \rightarrow \infty,
$$


such that $\forall x \in \mathbb{T} \backslash A n$ :

$$
\begin{gathered}
\exists L \in \mathbb{N} ; \\
M_{L+q_{n}}(x, \lambda, E)=M_{q_{n}}(0, \lambda, E) M_{L}(x, \lambda, E) \text { with } q_{n} \leqq L \leqq 4 q_{n} .
\end{gathered}
$$

Lemma 5. For every irrational $\alpha$ and for each energy $E \in B_{\infty}$,

$$
\lim _{n \rightarrow \infty} \frac{1}{q_{n}} \ln \left\|M_{(n)}\right\|=0 .
$$

The proof of (22) goes as follows: suppose that there exists an energy $E \in\{E ; \gamma(E)>0\} \cap B_{\infty}$.

Let now $\varepsilon>0$ be fixed and $N$ sufficiently large.

$$
\text { For } x \in\left(\mathbb{T} \backslash \bigcup_{n=N}^{\infty} A n\right) \cap \Omega_{0},
$$

we know that $\forall L^{\prime}>N(x, \varepsilon)(23)$ holds.

On the other hand if $n$ is large enough (say $q_{n}>N(x, \varepsilon)$ ) the above lemmas imply the existence of $L$ satisfying $q_{n} \leqq L \leqq 4 q_{n}$ and

$$
e^{(\gamma(E)-\varepsilon)\left(L+q_{n}\right)} \leqq\left\|M_{L+q_{n}}(x, \lambda, E)\right\| \leqq\left\|M_{q_{n}}(0, \lambda, E)\right\|\left\|M_{L}(x, \lambda, E)\right\| \leqq e^{\gamma_{n} q_{n}} e^{(\gamma(E)+\varepsilon) L} .
$$

Thus

$$
\gamma(E) q_{n} \leqq \gamma_{n} q_{n}+2 \varepsilon L+\varepsilon q_{n} \leqq\left(\gamma_{n}+9 \varepsilon\right) q_{n},
$$

since $\varepsilon$ is arbitrarily small and $\gamma_{n} \rightarrow 0$ as $n \rightarrow \infty$ we get a contradiction with $\gamma(E)>0$. So

$$
B_{\infty} \subset\{E \in \mathbb{R} ; \gamma(E)=0\} \text {. }
$$

Proof of Lemma 4. Let $R_{\alpha}: \mathbb{T} \rightarrow \mathbb{T}$ be the rotation of $\alpha: R_{\alpha}(x)=x+\alpha$ and let $\|x-y\|$ be the distance between $x$ and $y$ on the torus that is $\|x\| \equiv \inf _{p \in \mathbb{Z}}|x+p|$.

We have:

\section{Lemma 6.}

$$
\forall \alpha \in \mathbb{T}, \quad \forall M \in \mathbb{N}, \quad \forall \delta \in \mathbb{R},
$$

if we define

$$
A(\delta, \alpha, M) \equiv \bigcup_{i=0}^{M} R_{\alpha}^{-i}([-\delta, \delta] \cup[-\alpha-\delta,-\alpha+\delta])
$$

then:

$$
\begin{gathered}
\forall x \in \mathbb{T} \backslash A(\delta, A, M), \forall y ; \quad\|x-y\|<\delta \\
v(x+l \alpha)=v(y+l \alpha) \quad \forall l \in[0, M]
\end{gathered}
$$

and $|A(\delta, \alpha, M)| \leqq M \cdot 4 \delta$.

Assuming Lemma 6 we complete the proof of Lemma 4.

For a fixed irrational $\alpha$ and for $\delta, M$ and $x \in \mathbb{T} \backslash A(\delta, \alpha, M)$ fixed, by the ergodicity of $R_{\alpha}$, there exists an integer $N(\delta, \alpha, x)$ sufficiently large such that $\|x-N(\delta, \alpha, x) \alpha\|<\delta$. 
By using Lemma 6 the sequences of values of the potential starting from $x$ or from $N(\delta, \alpha, x) \alpha$ are equal for a length $M$.

Thus in order to study the sequence of potential starting from $x$ we can consider the history starting from zero and with a shift $N(\delta, \alpha, x)$.

For the trajectory starting from zero we have the recursion relations on the transfer matrices:

$$
M_{(n+1)} \equiv M_{q_{n+1}}(0, E, \lambda)=M_{(n-1)} M_{(n)}^{a_{n+1}}
$$

This means that for each $n$ we can assemble the infinite product of transfer matrices in products of blocks $M_{(n)}$ and $M_{(n-1)}$; in fact for any $l$ arbitrarily large $M_{(l)}$ can be written as a series of products of matrices $M_{(n)}$ and $M_{(n-1)}$,

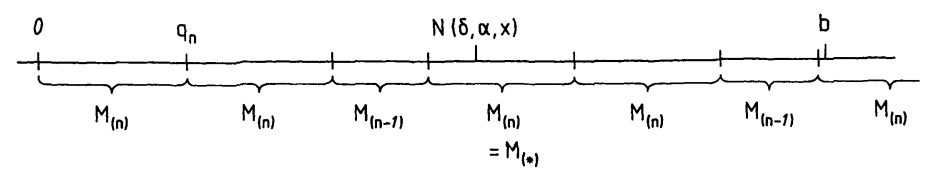

Fig. 1

In other words for each $n$ we can consider a tiling of the lattice with only two types of tiles of length $q_{n}$ or $q_{n-1}$. By the recursive relations of the matrices $M_{(n)}$ we know that blocks of type $M_{(n-1)}$ are separated one from the other by a sequence of blocks $M_{(n)}$ of length $a_{n+1}$ or $a_{n+1}+1$.

Let us now consider the block, of type $M_{(n)}$ or $M_{(n-1)}$, in which the point $N(\delta, \alpha, x)$ falls, say $M_{(*)}$, and let $b \in \mathbb{Z}$ be the first point of the second block of type $M_{(n)}$ on the right-hand side of $M_{(*)}$ (see Fig. 1).

Let $L=b-N(\delta, \alpha, x)$, by the definition of $b, q_{n}<L$ in fact $b$ is the starting point of the second block of type $M_{(n)}$ on the right-hand side of $M_{(*)}$, that is between $N(\delta, \alpha, x)$ and $b$ there will be surely another block $M_{(n)}$. On the other hand $L<2 q_{n}$ $+2 q_{n-1}$ because each block of type $M_{(n-1)}$ is isolated and so the longest string between $M_{(*)}$ and $b$ would be

$$
M_{(*)} M_{(n-1)} M_{(n)} M_{(n-1)}
$$

(remark that here we have used the order from left to right which would not be correct for matrices).

By the definition of $b$ and by Lemma 6 with

$$
\begin{aligned}
M & =5 q_{n}, \\
\delta & =\frac{1}{q_{n}^{2}},
\end{aligned}
$$

we have

$$
\begin{aligned}
M_{L+q_{n}}(x, \lambda, E) & =M_{L+q_{n}}(N(\delta, \alpha, x) \alpha, \lambda, E)=M_{(n)} M_{L}(N(\delta, \alpha, x) \alpha, \lambda, E) \\
& \left.=M_{q_{n}}(0, \lambda, E) M_{L}(x, \lambda, E)\right) .
\end{aligned}
$$


This means that if we choose the set $A_{n}$ as the set $A(\delta, \alpha, M)$ of Lemma 6 with the previous choice of $M$ and $\delta$ we have

$$
\sum_{n=N}^{\infty}\left|A_{n}\right| \leqq \sum_{n=N}^{\infty} \frac{20}{q_{n}} \leqq 20 \sum_{n=N}^{\infty} \frac{1}{2^{n / 2}}=20 \frac{\left(\frac{1}{\sqrt{2}}\right)^{N}}{1-\frac{1}{\sqrt{2}}} \rightarrow 0 \text { as } N \rightarrow \infty,
$$

and the lemma is proved.

Proof of Lemma 5. Let

$$
\gamma_{i}=\frac{1}{q_{i}} \log \left\|M_{(i)}\right\|
$$

By using the recursive relation and Lemma 2 we have

$$
\begin{aligned}
\left\|M_{(n+1)}\right\| & =\left\|M_{(n-1)} M_{(n)} \cdot S_{a_{n+1}-1}\left(\operatorname{tr} M_{(n)}\right)-M_{(n-1)} S_{a_{n+1}-2}\left(\operatorname{tr} M_{(n)}\right)\right\| \\
& \leqq\left\|M_{(n-1)}\right\|\left\|M_{(n)}\right\|\left|S_{a_{n+1}-1}\left(\operatorname{tr} M_{(n)}\right)\right|+\left\|M_{(n-1)}\right\|\left|S_{a_{n+1}-2}\left(\operatorname{tr} M_{(n)}\right)\right| \\
& \leqq e^{\gamma_{n-1} q_{n-1}} e^{\gamma_{n} q_{n}} C^{a_{n+1}}+e^{\gamma_{n-1} q_{n-1}} C^{a_{n+1}-1} \\
& \leqq 2 e^{\gamma_{n-1} q_{n-1}} e^{\gamma_{n} q_{n}} C^{a_{n+1}} .
\end{aligned}
$$

Since by Lemma $2\left|S_{a}(\xi)\right| \leqq a+1$ if $|\xi| \leqq 2$, and $\left|S_{a}(\xi)\right| \leqq|\xi|^{a+1}$ if $|\xi|>2$, and by Proposition 4 if $\left\{\left|\xi_{n}\right|\right\}$ is bounded then $\left|\xi_{n}\right|<2+\sqrt{ } 8+\lambda^{2} \equiv C$.

Thus

$$
\gamma_{n+1} \leqq \gamma_{n-1} \frac{q_{n-1}}{q_{n+1}}+\gamma_{n} \frac{q_{n}}{q_{n+1}}+\ln c \frac{a_{n+1}}{q_{n+1}}+\frac{\ln 2}{q_{n+1}}
$$

If $a_{n+1}>1$,

$$
\gamma_{n+1} \leqq \max \left\{\gamma_{n-1}, \gamma_{n}\right\} \frac{2}{3}+\frac{C^{\prime}}{q_{n}}
$$

In fact

$$
\frac{q_{n}+q_{n-1}}{a_{n+1} q_{n}+q_{n 1}}=\frac{1+\frac{q_{n-1}}{q_{n}}}{a_{n+1}+\frac{q_{n-1}}{q_{n}}}<\frac{2}{a_{n+1}+1}<\frac{2}{3}
$$

since $\frac{1+x}{a_{n+1}+x}$ is a monotone increasing function for $x \in(0,1)$.

If $a_{n+1}=1$,

$$
\left\|M_{(n+1)}\right\|=\left\|M_{(n-1)} M_{(n)}\right\|=\left\|M_{(n-1)} M_{(n-2)} M_{(n+1)}^{a n}\right\| .
$$

If $a_{n}>1$ the previous argument can be repeated to obtain:

$$
\gamma_{n+1} \leqq \gamma_{n-1} 2 \frac{q_{n-1}}{q_{n+1}}+\gamma_{n-2} \frac{q_{n-2}}{q_{n+1}}+\frac{C^{\prime}}{q_{n+1}} \leqq \max \left\{\gamma_{n-1}, \gamma_{n-2}\right\} \frac{2 q_{n-1}+q_{n-2}}{q_{n+1}}+\frac{C^{\prime}}{q_{n+1}},
$$


and

$$
\frac{2 q_{n-1}+q_{n-2}}{a_{n} q_{n-1}+q_{n-1}+q_{n-2}}=\frac{2+\frac{q_{n-2}}{q_{n-1}}}{a_{n}+1+\frac{q_{n-2}}{q_{n-1}}} \leqq \frac{3}{4} .
$$

If also $a_{n}=1$ we have to consider the previous scales:

$$
\left\|M_{(n+1)}\right\|=\left\|M_{(n-1)} M_{(n-2)} M_{(n-1)}\right\|=\left\|M_{(n-3)} M_{(n-2)}^{a_{n-1}} M_{(n-2)} M_{(n-3)} M_{(n-2)}^{a_{n-1}}\right\|,
$$

applying always the same argument we have:

$$
\left\|M_{(n+1)}\right\| \leqq e^{\gamma_{n-3} 2 q_{n-3}} e^{\gamma_{n-2} 2 q_{n-2}} 2 C^{a_{n-1}+1} 2 C^{a_{n-1}},
$$

that is:

$$
\gamma_{n+1} \leqq \max \left\{\gamma_{n-2}, \gamma_{n-3}\right\} \frac{2 q_{n-3}+2 q_{n-2}}{q_{n+1}}+\frac{C^{\prime}}{q_{n+1}}
$$

and

$$
\begin{aligned}
\frac{2 q_{n-3}+2 q_{n-2}}{q_{n+1}} & =\frac{2 q_{n-3}+2 q_{n-2}}{2 q_{n-1}+q_{n-2}}+\frac{2\left(q_{n-3}+2 q_{n-2}\right)}{2\left(a_{n-1} q_{n-2}+q_{n-3}\right)+q_{n-2}} \\
& =\frac{\frac{q_{n-3}}{q_{n-2}}+1}{\left(a_{n-1}+\frac{1}{2}+\frac{q_{n-3}}{q_{n-2}}\right)} \leqq \frac{1+x}{3} \text { with } \quad x \in(0,1),
\end{aligned}
$$

which is maximum for $x=1$ that is $\leqq \frac{4}{5}$. Thus $\gamma_{n} \rightarrow 0$ as $n \rightarrow \infty$, and thus the lemma is proved.

Proof of Lemma 6. By definition two points at a distance $\delta$, which obviously are always at the same distance under successive rotations, have a different history only if they or their evolutions under $R_{\alpha}$ fall in $[-\delta, \delta] \cup[-\alpha-\delta$, $-\alpha+\delta]$. But the set $A(\delta, \alpha, A)$ is constructed explicitly in order to avoid this on a sequence of length $M$.

\section{Remarks and Open Problems}

In this final section we want to point out some open problems.

1. We have considered in this paper the special model (1), (2) with the particular choice of the interval $I:[1-\alpha, 1[$. First of all we want to remark that our choice is essentially identical to that of all the people working in the golden case, in fact for the golden number $\alpha^{2}+\alpha=1$.

Different choices of the interval $I$ would be possible (see for instance [Be.Sc.]) and different choices of the potential as well, but the recursive relations could become much more difficult.

2. This proof of Cantor spectrum is not constructive as in the paper by Casdagli and as it would be expected to be by numerical results, and thus the 
problem of the control of the Hausdorff dimension of the spectrum is completely open. We believe that an alternative, more constructive proof, can be done in the same generality as the proof given in this paper.

3. As far as the nature of the spectrum is concerned, as mentioned in the introduction, we cannot conclude that the spectrum is singular continuous for all $x \in \mathbb{T}$ since the problem of the extension of the proof of the absence of localization to all $x \in \mathbb{T}$ is still open.

4. The study of the spectrum as a set does not give a complete physical description of the model. One can look, for instance, at the diffusion properties of the model, that is at the evolution of a wave packet. We want to emphasize here that the diffusion properties can be very peculiar also in cases in which the nature of the spectrum is known. We mention, as an example, the hierarchical case [J.-L., Ma.Sc.] where the spectrum turns out to be singular and with a Cantor structure, and the diffusion has a logarithmic low.

5. Always in the one dimensional case, one can look at other almost periodic problems. The simplest example can be done by the Laplace operator, mentioned in the introduction, defined on the almost periodic lattice [Lu.Pe.] which, by a suitable change of variables can be reduced to our model. The eigenvalue problem for the original model is not completely solved from a rigorous point of view, (see [Le]).

Acknowledgements. Very special thanks are due to F. Martinelli for his crucial and timely suggestion to apply the Kotani result, and for the many useful discussions both on this and on other related problems. We wish to thank L. S. Levitov and A. Süto for useful discussions on the model studied in this paper. One of us [E.S.] wishes to thank the Université de Provence, Marseille and the Centre de Physique Theorique CNRS, Luminy for the kind hospitality during the preparation of the paper and A. Klein for his kind invitation to visit the Department of Mathematics of University of California-Irvine, where part of this work has been done.

When this paper was completed, we received a preprint by Süto on a rigorous proof of Cantor spectrum in the particular case of the golden number [Su2].

\section{References}

[Al]

[A.A] Aubry, S., Andre, G.: Analyticity breaking and the Anderson localization in incommensurate lattices. Ann. Israel Phys. Soc. 3, 133-164 (1980)

[A.A.K.M-f, P] Axel, F., Allouche, J.P., Kleman, M., Mendes-France, M., Peyriere, J.: Vibrational modes in a one dimensional "quasi-alloy": the Morse case. J. Phs. C3, 47, C3 181-C3 186 (1986)

[B.G.H] Bransley, M.F., Geronimo, J.S., Harrington, A.N.: Almost periodic Jacobi matrices associated with Julia sets for polynomials. Commun. Math. Phys. 99, 303-317 (1985)

[Be] Bellissard, J.: Almost periodicity in solid state physics and $c^{*}$ algebras, H. Bohr Centennary Conference on almost periodic functions, to appear (1987)

[Be.Bes.Mou.] Bellissard, J., Bessis, D., Moussa, P.: Chaotic states of almost periodic Schrödinger operators. Phys. Rev. Lett. 49, 701-704 (1982)

[Be.Sc] Bellissard, J., Scoppola, E.: The density of states for almost periodic Schrödinger operators and the frequency module: a counterexample. Commun. Math. Phys. 85, 301-308 (1982) 
[Bou.Lac] Bougerol, Ph., Lacroix, J.: Products of random matrices with applications to Schrödinger operators. Boston, Stuttgart: Birkhäuser

[Ca] Casdagli, M.: Symbolic dynamics for the renormalization map of a quasiperiodic Schrödinger equation. Commun. Math. Phys. 107, 295 (1986)

[De.Pe] Delyon, F., Petritis, D.: Absence of localization in a class of Schrödinger operators with quasiperiodic potential. Commun. Math. Phys. 103, 441 (1986)

[G.W.R.P.B] Ghez, J.M., Wang, W., Rammal, R., Pannetier, B., Bellissard, J.: Band spectrum for an electron on a Serpinsky gasket in a magnetic field. Sol. State Commun. 64, 1291-1294 (1987)

[G.A] Gumbs, G., Ali, M.K.: Scaling and eigenstates for a class of one dimensional quasi-periodic lattices. J. Phys. A 21, L517-L521 (1988)

[G.A1] Gumbs, G., Ali, M.K.: Dynamical maps, Cantor spectra, and localization for Fibonacci and related quasiperiodic lattices. Phys. Rev. Lett. 60, 1081-1084 (1988)

[H.K] Huberman, B.A., Kerzberg, M.: Ultra-diffusion: the relaxation of hierarchical systems. J. Phys. A 18, L331-L 336 (1985)

[J.D] Janot, Ch., Dubois, J.M.: Editors: Quasicrystalline materials. Grenoble 21-25 march 1988. Singapore: World Scientific 1988

[J.-L.Ma.Sc] Jona-Lasinio, G., Martinelli, F., Scoppola, E.: Multiple tunneling in $d$-dimensions: a quantum particle in a hierarchical potential. Ann. Inst. Henri Poincaré 42, 73-108 (1985)

[Ka.Kom.Ta] Kadanoff, L.P., Kohmoto, M., Tang, C.: Localization problem in one dimension: mapping and escape. Phys. Rev. Lett. 50, 1870-1872 (1983)

[Kal.Ki.Le] Kalugin, P.A., Kilaev, A.Yu., Levitov, S.: Electron spectrum of a one dimensional quasi-crystal. Sov. Phys. JETP 64, 410-415 (1986)

[K] Komoto, M.: Metal insulator transition and scaling for incommensurate system. Phys. Rev. Lett. 51, 1198-1201 (1983)

[K.B] Komoto, M., Banavar, J.R.: Quasi-periodic lattice: electronic properties and diffusion, Phys. B 34, 563-566 (1986)

[Ko] Kotani, S.: Jacobi matrices with random potentials taking finitely many values, Preprint Tokyo (1989)

[K.L.S] Kunz, H., Livi, R., Suto, A.: Cantor spectrum and singular continuity for a hierarchical hamiltonian. Commun. Math. Phys. 122, 643-679 (1989)

[La] Lang, S.: Introduction to diophantine approximations, Reading MA; AddisonWesley, 1966

[Le] Levitov, L.S.: Renormalization group for a quasiperiodic Schrödinger operator. J. Stat. Phys. (to appear)

[Lu] Luck, J.M.: Cantor spectra and scaling of gap widths in deterministic aperiodic systems. Phys. Rev. (to appear)

[Lu.Pe] Luck, J.M., Petritis, D.: Phonon spectra in one-dimensional quasicrystal. J. Stat. Phys. 42, 289-310 (1986)

[Ma.Na] Machida, K., Nakano, M.: Soliton lattice structure and mid-gap band in nearly commensurate charge-density-wave states. II Self-similar band structure and coupling constant dependence. Phys. Rev. B 34, 5073-5081 (1986)

[Ma.Sc] Martinelli, F., Scoppola, E.: Introduction to the mathematical theory of Anderson localization. Rivista del Nuovo Cimento 10 (1987)

[Os.Kim] Ostlund, S., Kim, S.: Renormalization of quasi periodic mappings. Physica Scripta 9, 193-198 (1985)

[O.P.R.S.S] Ostlund, S., Prandit, R., Rand, D., Schnellnhuber, H.J., Siggia, E.D.: One dimensional Schrödinger equation with an almost periodic potential. Phys. Rev. Lett. 50, 1873-1877 (1983)

[Ra] Rammal, R.: Spectrum of harmonic excitations on fractals. J. Phys. 45, 191-206 (1984)

[R.L.T] Rammal, R., Lubensky, T.C., Toulouse, G.: Supraconducting networks in a magnetic field. Phys. Rev. B 27, 2820-2829 (1983) 
[R.O.S.S] Rand, D., Ostlund, S., Sethna, J., Siggia, E.D.: Universal transition from quasi periodicity to chaos in dissipative systems. Phys. Rev. Lett. 49, 132-135 (1982)

[Re.Si] Reed, M., Simon, B.: Methods of modern mathematical physics. I. Functional analysis. New York: Academic Press 1972

[Sh.Bl.Gr.Ca] Schechtman, D., Blech, I., Gratias, D., Cahn, J.V.: Metallic phase with long range orientational order and no translational symmetry. Phys. Rev. Lett. 53, 1951-1953 (1984)

[Si] Simon, B.: Almost periodic Schrödinger operators: A review. Adv. Appl. Math. 3, 463-490 (1982)

[S.O] Steinhardt, P.J., Ostlund, S.: The physics of quasicrystals. Singapore: World Scientific 1987

[S.K] Sutherland, B., Kohmoto, M.: Resistance of a one dimensional quasi crystal: power law growth. Phys. Rev. B 36, 5877-5886 (1987)

[Sü] Süto, A.: The spectrum of a quasi-periodic Schrödinger operator. Commun. Math. Phys. 111, 409-415 (1987)

[Sü2] Süto, A.: Singular continuous spectrum on a Cantor set of zero Lebesgue measure for the Fibonacci Hamiltonian. J. Stat. Phys. (to appear)

Communicated by B. Simon

Received March 7, 1989 
